\title{
T-DUALITY INVARIANCE IN RANDOM LATTICE STRINGS
}

\author{
W. Siegel ${ }^{1}$ \\ Institute for Theoretical Physics \\ State University of New York, Stony Brook, NY 11794-3840
}

\begin{abstract}
Preserving the T-duality invariance of the continuum string in its random lattice regularization uniquely determines the random matrix model potential. For $\mathrm{D}=0$ the duality transformation can be performed explicitly on the matrix action, and replaces color with flavor; invariance thus requires that the color and flavor groups be the same.
\end{abstract}

1 Internet address: siegel@insti.physics.sunysb.edu. 


\section{INTRODUCTION}

By definition renormalization must preserve as many properties as possible of a classical field theory. In particular this includes a maximal set of nonanomalous global or local symmetries. The simplest way to renormalize consistently with this requirement is to use a regularization scheme that automatically preserves as many as possible of these symmetries, so as to avoid the inconvenience of finite renormalizations and Ward-Takahashi-Slavnov-Taylor identities. Regularization schemes that preserve symmetries (such as dimensional regularization) also tend to make calculations simpler.

One regularization scheme that has the added benefit of providing a calculational scheme for nonperturbative quantum field theory is the use of a lattice. In the case of strings, the worldsheet is replaced with a lattice, whose two-dimensional nature is preserved by the topological properties of the $1 / N$ expansion, and whose arbitrary worldsheet metric is manifested through the randomness of the Feynman diagrams that represent the lattices [1]. These Feynman diagrams belong to an underlying field theory whose bound states are strings. Generally the continuum limit, which is the limit of vanishing worldsheet cosmological constant, also requires the limit of infinite colors $(N)$, as a result of dimensional transmutation. However, the "lumpy" string that results from fixed $N$ may be of more physical relevance, and can be treated by expansion about the continuum string.

The fact that this lattice replaces the worldsheet, and not physical spacetime (as in lattice QCD), means that spacetime symmetries, such as Poincaré invariance, can be manifestly preserved by this regularization. However, one spacetime symmetry of (closed) string theory that has been completely ignored on the worldsheet lattice is Tduality invariance [2]: Although T-duality transformations on random lattices have been considered [3], the fact that the continuum string theory is invariant under this transformation has never been used to define the lattice regularization of the worldsheet. By T-duality we refer here to the transformation $\partial_{m} x=\epsilon_{m n} \partial^{n} x^{\prime}$; i.e., Hodge duality on $\partial x$. In this paper T-duality will be applied to the underlying fundamental fields of the random matrix model, and not just the composite fields of the string theory, whose T-duality transformations are commonly studied through the introduction of background fields.

Since neither the regularization nor the renormalization of these matrix models has been shown to preserve T-duality invariance, it is not clear whether spurious terms might have been introduced into the path integral, in the same way that using 
a momentum-space cutoff for QCD produces terms that aren't gauge invariant. Although such terms might be avoided in lower dimensions, universality is not expected in more-physical cases. Also, we may want to preserve symmetries for finite $N$. In this paper we show that duality invariance uniquely determines the lattice regularization by fixing the matrix model potential, and find the resulting model. At least in $\mathrm{D}=0$, this model of "gluons" (hermitian matrices) has a natural interpretation in terms of a theory of self-interacting bosonic "quarks" (complex matrices), whose action has just quadratic and quartic terms.

In $\mathrm{D}=0$ the only effect of the duality transformation is to replace a Feynman graph with the dual graph. We show how to explictly perform this transformation on the matrix model action. Duality switches the color and flavor of the quarks, so duality invariance requires the equality of the color and flavor groups.

\section{THE MODEL}

A T-duality transformation on a quantum field theory, as defined by treating that theory as the underlying theory of a string theory, has two main effects on the Feynman diagrams of that field theory [4]: (1) It replaces the propagator with its Fourier transform. This is a trivial invariance for the usual strings, where these propagators are Gaussians. (However, in more-realisitic field theories, with $1 / p^{2}$ propagators, this is an invariance only in $\mathrm{D}=4$ [5].) (2) It replaces the Feynman diagram (lattice) with the dual lattice - the graph resulting from replacing vertices with loops (faces) and vice versa. This is the lattice version of Hodge duality.

Invariance of the theory under replacement of a diagram with its dual diagram not only requires that both diagrams actually exist as Feynman diagrams of that theory, but also that the weights of those two diagrams be identical. The symmetries of two dual diagrams are the same, so they have identical combinatoric factors; thus we need to compare only the products of their coupling constants. Duality relates diagrams that not only have different numbers of vertices, but also vertices with different numbers of lines, so it relates the different n-point couplings. Since any theory has diagrams with loops with an arbitrary number of sides, the dual diagrams will have vertices with an arbitrary number of lines, so the potential must be nonpolynomial.

It is simplest to consider vacuum graphs, and the duality of the corresponding polyhedra (which approximate the sphere). The "watermelon" graph consisting of $\mathrm{n}$ 
propagators connecting two n-point vertices is dual to a single loop with n two-point vertices. From this we find

$$
N^{n}\left(g_{n}\right)^{2}=N^{2}\left(g_{2}\right)^{n} \Rightarrow\left|\frac{g_{n}}{N}\right|=\left|\frac{g_{1}}{N}\right|^{n}
$$

which is sufficient to determine all couplings in terms of the one-point coupling, up to signs. (In particular, it tells us $g_{1}$ is nonvanishing.) The "daisy" graph, consisting of a single 2n-point vertex with $\mathrm{n}$ loops, is dual to the "pincushion" tree graph, consisting of one $n$-point vertex whose legs are terminated by $n$ one-point vertices. This gives the relation

$$
N^{n+1} g_{2 n}=N g_{n}\left(g_{1}\right)^{n} \Rightarrow \frac{g_{2 n}}{N}=\frac{g_{n}}{N}\left(\frac{g_{1}}{N}\right)^{n}
$$

which determines all even-point vertices from lower-point ones: e.g., $g_{2} / N=\left(g_{1} / N\right)^{2}$, $g_{4} / N=\left(g_{1} / N\right)^{4}$. Replacing one "petal" of the daisy with a "pin" does the opposite on the dual graph, giving the relation

$$
N^{n+1} g_{2 n+1} g_{1}=N^{2} g_{n+2}\left(g_{1}\right)^{n} \Rightarrow \frac{g_{2 n+1}}{N}=\frac{g_{n+2}}{N}\left(\frac{g_{1}}{N}\right)^{n-1}
$$

which determines all odd-point vertices except $g_{3}$ from lower-point ones. Finally, the lollipop graph (loop with a stem) with a two-point vertex on the loop is dual to the figure-eight graph with a two-point vertex on one loop, so

$$
N^{2} g_{2} g_{4}=N^{3} g_{1} g_{2} g_{3} \quad \Rightarrow \quad \frac{g_{3}}{N}=\frac{g_{4}}{N}\left(\frac{g_{1}}{N}\right)^{-1}
$$

These diagrams are thus sufficient to solve for all vertices in terms of the one-point vertex. The unique solution for the n-point couplings is then

$$
g_{n}=N g^{n}
$$

in terms of a single coupling $g$.

One might worry that duality for all Feynman graphs would overdetermine these couplings, but the identity (from counting ends of all lines)

$$
\sum n V_{n}=2 P-E
$$

for $V_{n}$ n-point vertices, $P$ propagators, and $E$ external lines shows that $\sum n V_{n}$ is an invariant under duality (since $P$ and $E$ are). Thus in vacuum graphs $g$ always appears as $g^{2 P}$ : While usually either the total number of vertices $V\left(=\sum V_{n}\right)$ or the number of loops $L$ is used to define the "area" of the lattice, the duality invariant definition 
is to use something proportional to their average, which follows here from the usual $\hbar$-counting identity

$$
P-V=L-1 \quad \Rightarrow \quad P=V+L-1
$$

The potential for the hermitian $N \times N$ matrix field $\phi$ appearing in $e^{-S}$ is then

$$
-N \sum_{n=1}^{\infty} \frac{1}{n} g^{n} \phi^{n}=N \ln (1-g \phi)
$$

(The combinatoric factor is $1 / n$ and not $1 / n$ ! because we consider only planar diagrams, so lines from a vertex are ordered.) This result can also be obtained as a special case of the work of [6], where general weights for both vertices and loops were considered, by restricting their potential to be $\mathrm{U}(N)$ invariant and have equal couplings for vertices and loops.

This gives the action

$$
S=\operatorname{tr} \int \frac{d^{D} x}{(2 \pi)^{D / 2}}\left[\frac{1}{2} \phi e^{-\square / 2} \phi+N \ln (1-g \phi)\right]
$$

If we rescale $\phi \rightarrow \phi / g$ we can associate the coupling with the kinetic term:

$$
S^{\prime}=\operatorname{tr} \int \frac{d^{D} x}{(2 \pi)^{D / 2}}\left[\frac{1}{2 g^{2}} \phi e^{-\square / 2} \phi+N \ln (1-\phi)\right]
$$

along with a rescaling of the functional measure. This gives the above counting of $g$ 's in terms of propagators. The form appropriate for the $1 / N$ expansion is obtained by further rescaling $g \rightarrow g / \sqrt{N}$ :

$$
S^{\prime \prime}=N \operatorname{tr} \int \frac{d^{D} x}{(2 \pi)^{D / 2}}\left[\frac{1}{2 g^{2}} \phi e^{-\square / 2} \phi+\ln (1-\phi)\right]
$$

This model was considered in [7]. It is related to the Penner model [8], except that here (the exponential of) the worldsheet cosmological constant modifies the kinetic term rather than the potential.

\section{DUALIZATION OF RANDOM MATRIX FIELDS}

We now specialize to the simplest case, $\mathrm{D}=0$. Then

$$
S_{\phi}=\operatorname{tr}\left[\frac{1}{2} \phi^{2}+N \ln (1-g \phi)\right]
$$

with the unitary "color" symmetry

$$
\phi \rightarrow U_{c} \phi U_{c}^{-1}
$$


The logarithmic term suggests its derivation from bosonic "quarks", in addition to the scalar "gluons" $\phi$. This gives a more natural-looking form for the action that is real for all values of the fields:

$$
S_{\psi \phi}=\operatorname{tr}\left[\frac{1}{2} \phi^{2}+\psi^{\dagger}(1-g \phi) \psi\right]
$$

for the complex $(N \times N)$ matrix $\psi$ with $N$ colors and $N$ flavors, which gives the previous action as an exact result after Gaussian integration. The quark field transforms under both color and flavor symmetries

$$
\psi \rightarrow U_{c} \psi U_{f}^{-1}
$$

Unlike QCD, this $1 / N$ expansion with gluons and quarks describes only closed strings and not open, since in models with the number of flavors proportional to the number of colors the topological expansion is identical to that of pure Yang-Mills theory. The action $S_{\psi \phi}$ actually somewhat resembles 2D QCD [9] in that there is no gluon self-interaction.

We can instead perform the $\phi$ integration on $S_{\psi \phi}$, which is now also Gaussian, to find

$$
S_{\psi}=\operatorname{tr}\left[\psi^{\dagger} \psi-\frac{1}{2} g^{2}\left(\psi^{\dagger} \psi\right)^{2}\right]
$$

The quartic term has the wrong sign, just as in the usual $\phi^{4}$ matrix model of strings. Such models have been studied in $[10,11]$, and shown to give results similar to those of hermitian matrix models.

This action can in turn be derived from an action similar to $S_{\psi \phi}$, but with $\phi$ replaced by a flavor field $\chi$ :

$$
S_{\psi \chi}=\operatorname{tr}\left[\frac{1}{2} \chi^{2}+\psi(1-g \chi) \psi^{\dagger}\right]
$$

where the new field transforms under only the flavor symmetry

$$
\chi \rightarrow U_{f} \chi U_{f}^{-1}
$$

Finally, we reverse the initial step by integrating out $\psi$ to obtain an action identical in form to the original one $S_{\phi}$ :

$$
S_{\chi}=\operatorname{tr}\left[\frac{1}{2} \chi^{2}+N \ln (1-g \chi)\right]
$$

The most interesting thing about these transformations is that they explicitly perform a duality transformation on the matrix model action. To relate to the usual 
definition of T-duality in terms of Feynman graphs, we examine the effects of these field transformations on the diagrams, and observe that they explicitly perform a construction that replaces a graph with the dual graph, where the original graph is drawn with color lines, while the dualized graph is drawn with flavor lines. As usual, each propagator is drawn with a double line, each line representing an index on the matrix, and the continuity of the lines representing the (unitary) symmetries. The planar graphs are those in which no lines cross, and all external lines are on the outside of the graph. (Actually, we here consider vacuum graphs drawn on the sphere.) The original graph is one following from $S_{\phi}$. The equivalent graph from $S_{\psi \phi}$ results from inserting a (one-line) flavor loop into each vertex. Eliminating $\phi$ to obtain $S_{\psi}$ has the effect of contracting the $\phi$ propagators to points. Then introducing $\chi$ to obtain $S_{\psi \chi}$ causes $\chi$ propagators to expand the $\psi$ four-point vertices. Thus the change from $S_{\psi \phi}$ to $S_{\psi \chi}$ has replaced all $\phi$ propagators (double color lines) with $\chi$ propagators (double flavor lines) pointing in the orthogonal direction. Finally, eliminating $\psi$ to obtain $S_{\chi}$ simply shrinks all the color loops to points. Thus all color vertices have been replaced with flavor loops, all color loops have been replaced with flavor vertices, and all color propagators have been replaced with orthogonal flavor propagators. So, switching from color to flavor is exactly a duality transformation.

We can also consider more general models, using the same form of action as $S_{\psi}$, with $N$ colors but with a different number $M$ of flavors [10,11]. Such a theory is not duality invariant, but duality transforms a modified $S_{\phi}$

$$
S_{\phi}=\operatorname{tr}\left[\frac{1}{2} \phi^{2}+M \ln (1-g \phi)\right]
$$

for $N \times N \phi$ into a modified $S_{\chi}$ that is identical in form to the previous but with $\chi M \times M$. In particular, we can thus duality transform this $S_{\phi}$ for the case $M=1$ to an $S_{\chi}$ for a single variable $(1 \times 1$ matrix $) \chi$. Such "vector" models have been studied in $[12,11]$. The double-scaling limit used for the Penner model [13] and its generalizations kept $N-M$ fixed at a nonvanishing value, while effectively taking the limit of our $g \rightarrow \infty$, while in our case we must fix $N-M=0$ for duality invariance, and take a double-scaling limit where $g$ goes to a finite nonvanishing constant.

For $\mathrm{D}>0$ an auxiliary term for $\psi$ as in $S_{\psi \phi}$ generates a $\delta^{D}(0)$ coefficient for the logarithmic term, which violates duality, so the form of the duality transformation will require some generalization. 


\section{ACKNOWLEDGMENTS}

I thank Fabian Eßler for $N$ invaluable discussions. This work was supported in part by the National Science Foundation Grant No. PHY 9309888.

\section{REFERENCES}

[1] H.B. Nielsen and P. Olesen, Phys. Lett. 32B (1970) 203;

B. Sakita and M.A. Virasoro, Phys. Rev. Lett. 24 (1970) 1146;

G. 't Hooft, Nucl. Phys. B72 (1974) 461;

F. David, Nucl. Phys. B257 [FS14] (1985) 543;

V.A. Kazakov, I.K. Kostov and A.A. Migdal, Phys. Lett. 157B (1985) 295;

M.R. Douglas and S.H. Shenker, Nucl. Phys. B335 (1990) 635;

D.J. Gross and A.A. Migdal, Phys. Rev. Lett. 64 (1990) 127;

E. Brézin and V.A. Kazakov, Phys. Lett. 236B (1990) 144.

[2] W. Siegel, Phys. Lett. 134 (1984) 318;

T.H. Buscher, Phys. Lett. 194B (1987) 59, 201B (1988) 466.

[3] D.V. Boulatov, V.A. Kazakov, I.K. Kostov, and A.A. Migdal, Nucl. Phys. B275 [FS17] (1986) 641;

W. Siegel, Phys. Lett. 252B (1990) 558.

[4] F. David and R. Hong Tuan, Phys. Lett. 158B (1985) 435.

[5] W. Siegel, Actions for QCD-like strings, Stony Brook preprint ITP-SB-96-1 (January 1996), hep-th/9601002.

[6] V.A. Kazakov, M. Staudacher, and T. Wynter, LPTENS preprints 95-9, 95-24, 95-56, and 96-07 (hep-th/9502132, 9506174, 9601069, and 9601153).

[7] I.K. Kostov and M.L. Mehta, Phys. Lett. 189B (1987) 118.

[8] R.C.Penner, Bull.Am.Math.Soc. 15 (1986) 73, J.Diff.Geom. 27 (1988) 35;

J. Harer and D. Zagier, Invent. Math. 85 (1986) 457.

[9] G. 't Hooft, Nucl. Phys. B75 (1974) 461.

[10] T.R. Morris, Nucl. Phys. B356 (1991) 703;

J. Ambjørn, J. Jurkiewicz, and Yu.M. Makeenko, Phys. Lett. 251B (1990) 517.

[11] A. Anderson, R.F. Myers, and V. Periwal, Phys. Lett. 254B (1991) 89.

[12] S. Nishigaki and T. Yoneya, Nucl. Phys. B348 (1991) 787;

P. Di Vecchia, M. Kato, and N. Ohta, Nucl. Phys. B357 (1991) 495.

[13] J. Distler and C. Vafa, Mod. Phys. Lett. A6 (1991) 259. 